\title{
Intrinsic finite-energy Cooper pairing in $j=3 / 2$ superconductors
}

\author{
Masoud Bahari $\odot,{ }^{1,2, *}$ Song-Bo Zhang ${ }^{10},{ }^{1,2}$ and Björn Trauzettel ${ }^{1,2}$ \\ ${ }^{1}$ Institute for Theoretical Physics and Astrophysics, University of Würzburg, D-97074 Würzburg, Germany \\ ${ }^{2}$ Würzburg-Dresden Cluster of Excellence ct.qmat, Germany
}

(Received 3 August 2021; accepted 19 January 2022; published 11 February 2022)

\begin{abstract}
We show that Cooper pairing can occur intrinsically away from the Fermi surface in $j=3 / 2$ superconductors with strong spin-orbit coupling and equally curved bands in the normal state. In contrast to conventional pairing between spin- $1 / 2$ electrons, we derive that pairing can happen between interband electrons having different magnetic quantum numbers, for instance, $m_{j}=1 / 2$ and $m_{j}=3 / 2$. Such superconducting correlations manifest themselves by a pair of indirect gaplike structures at finite excitation energies. An observable signature of this exotic pairing is the emergence of a pair of symmetric superconducting coherence peaks in the density of states at finite energies. Moreover, the angular-momentum-resolved density of states in the presence of a perturbative Zeeman field reflects the $m_{j}$ composition of the Cooper pairs. We argue that such finite-energy pairing is a generic feature of $j=3 / 2$ superconductors, both in the presence and absence of inversion symmetry.
\end{abstract}

DOI: 10.1103/PhysRevResearch.4.L012017

Introduction. Since the discovery of the Bardeen-CooperSchrieffer theory for superconductivity [1], extensive efforts of theoretical and experimental research have been carried out to understand the pairing mechanism [2,3]. In most cases, superconductivity can be described by the pairing of spin$1 / 2$ electrons at the Fermi surface. However, it has been shown theoretically that the pairing of electrons with a higher total angular momentum is also possible [4-6]. This has triggered attempts to formulate a general theory of high angular momentum superconductivity [7-10] and to identify typical physical observables [11-22]. Prominent candidate materials for high angular momentum superconductivity are half-Heusler compounds whose Fermi surface lies close to the $\Gamma_{8}$ band with a total angular momentum quantum number $j=3 / 2$ [23-41]. These materials can be categorized into two distinct groups with inverted [23-36,38-42] and normal $[31,37,38,41]$ band structures, respectively. In the inverted case, only a single pair of $\Gamma_{8}$ bands with identical components of total angular momentum cross the Fermi energy [6,42$45]$. Despite the $j=3 / 2$ nature of the electrons, the pairing mechanism in this case can be captured within the formalism for (pseudo)spin-1/2 electrons at low energies [6]. In contrast, in the group with a normal band structure, density functional theory calculations predict that all $\Gamma_{8}$ bands bend downward near the Fermi energy [24,25,41,43-45]. This band structure applies, for instance, to $R \mathrm{PdBi}$ with $R \in\{\mathrm{Y}, \mathrm{Dy}, \mathrm{Tb}, \mathrm{Sm}\}$ $[31,37,38,41,46]$. We demonstrate below that such a configuration of energy bands in combination with superconductivity allow us to observe Cooper pairing composed by electrons

\footnotetext{
*masoud.bahari@physik.uni-wuerzburg.de

Published by the American Physical Society under the terms of the Creative Commons Attribution 4.0 International license. Further distribution of this work must maintain attribution to the author(s) and the published article's title, journal citation, and DOI.
}

with nonidentical magnetic quantum numbers $m_{j}$ at finite excitation energies (FEEs).

The pairing of spin-1/2 electrons with different orbitals at FEEs has been proposed for iron pnictides in the absence of spin-orbit coupling [47]. Recently, it has been argued that Ising superconductors may realize the finite-energy pairing of spin- $1 / 2$ electrons by applying external in-plane magnetic fields [48].

Hence, the question we address in this Letter is whether it is possible to observe intrinsic finite-energy Cooper pairing composed by electrons with different magnetic quantum numbers in the absence of any fields. We show below that the interplay of strong spin-orbit coupling and superconductivity allows for such pairing accompanied by a pair of indirect gaplike structures (GLSs) away from the Fermi energy. The electrons responsible for the finite-energy pairing originate from energy bands with different band indices. Our results suggest that such behavior is a generic feature of multiband superconductors when the $j=3 / 2$ electrons of the $\Gamma_{8}$ band contribute to pairing. In experiments, the GLSs manifest themselves by the appearance of a pair of symmetric superconducting coherence peaks at FEEs of the density of states (DOS). To elucidate that such Cooper pairing is a generic phenomenon of multiband superconductors preserving (breaking) inversion symmetry, we systematically analyze the role of $j=3 / 2$ pairing valid for cubic point group symmetry $O_{h}\left(T_{d}\right)$ based on the Luttinger-Kohn model.

Model. Low-energy $j=3 / 2$ electrons within the $\Gamma_{8}$ bands can be described by the $\mathbf{k} \cdot \mathbf{p}$ Luttinger-Kohn model $[49,50]$, $H_{0}=\sum_{\mathbf{k}} \hat{c}_{\mathbf{k}}^{\dagger} \hat{\mathcal{H}}_{0}(\mathbf{k}) \hat{c}_{\mathbf{k}}$, where

$$
\hat{\mathcal{H}}_{0}(\mathbf{k})=\alpha k^{2} \hat{I}_{4}+\beta \sum_{i} k_{i}^{2} \hat{J}_{i}^{2}+\gamma \sum_{i \neq j} k_{i} k_{j} \hat{J}_{i} \hat{J}_{j}-\mu \hat{I}_{4},
$$

and the basis is $\hat{c}_{\mathbf{k}}=\left(c_{\mathbf{k}, 3 / 2}, c_{\mathbf{k}, 1 / 2}, c_{\mathbf{k},-1 / 2}, c_{\mathbf{k},-3 / 2}\right)^{T}$. We denote $\mathbf{k}=\left(k_{x}, k_{y}, k_{z}\right)$ as the three-dimensional (3D) momentum, $k=|\mathbf{k}|, \hat{J}_{i}$ with $i \in\{x, y, z\}$ as the $4 \times 4$ total angular 


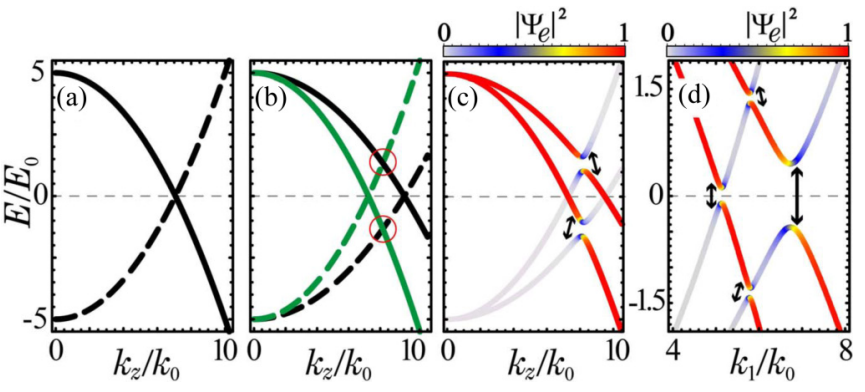

FIG. 1. BdG spectra along the $[0,0,1]$ direction in absence of pairing for (a) $\beta=0$ and (b) $\beta=0.2|\alpha|$, respectively. The spectra are independent of $\gamma$. BdG spectrum in the (c) $[0,0,1]$ and (d) $[1,1,0]$ (i.e., $k_{x}=k_{y}=k_{1}$ ) directions for $\gamma=\beta$ in the presence of septet pairing with amplitude $\Delta / E_{0} a=4.15$. The color denotes the probability of electronic states $\left|\Psi_{e}\right|^{2}$ in both (c) and (d). Other parameters are $\mu / E_{0}=-5, k_{0}=10^{-2} a^{-1}, E_{0}=10^{-3}|\alpha| a^{-2}$, and $\alpha=-20 . a$ is the lattice constant in a tight-binding version of the continuum model.

momentum matrices in the $j=3 / 2$ representation, and $\hat{I}_{4}$ as the $4 \times 4$ identity matrix. The material-dependent parameters $\alpha$ and $\beta(\gamma)$ control the kinetic energy and symmetric spin-orbit coupling, respectively; $\mu$ is the Fermi energy. The doubly degenerate eigenenergies of $\hat{\mathcal{H}}_{0}(\mathbf{k})$, protected by a combination of inversion and time-reversal symmetries, are given by

$$
E_{\mathbf{k}}^{ \pm}=\left(\alpha+\frac{5}{4} \beta\right) k^{2} \pm \beta \sqrt{\sum_{i}\left[k_{i}^{4}+\left(\frac{3 \gamma^{2}}{\beta^{2}}-1\right) k_{i}^{2} k_{i+1}^{2}\right]}-\mu,
$$

where $i+1=y$ if $i=x$ (notation used throughout this Letter). To investigate the properties of the excitation spectrum of Eq. (1) in the presence of high angular momentum Cooper pairing in $O_{h}$ symmetry, we introduce the full superconducting Hamiltonian given by $H=\sum_{\mathbf{k}} \hat{\psi}_{\mathbf{k}}^{\dagger} \hat{H}_{\mathrm{BdG}}(\mathbf{k}) \hat{\psi}_{\mathbf{k}}$, where $\hat{\psi}_{\mathbf{k}}=\left(\hat{c}_{\mathbf{k}}, \hat{c}_{-\mathbf{k}}^{\dagger T}\right)^{T}$ is the Nambu spinor. The Bogoliubov-de Gennes $(\mathrm{BdG})$ Hamiltonian takes the form

$$
\hat{H}_{\mathrm{BdG}}(\mathbf{k})=\left(\begin{array}{cc}
\hat{\mathcal{H}}_{0}(\mathbf{k}) & \hat{\mathcal{H}}_{\eta}^{J, S, L}(\mathbf{k}) \\
{\left[\hat{\mathcal{H}}_{\eta}^{J, S, L}(\mathbf{k})\right]^{\dagger}} & -\hat{\mathcal{H}}_{0}^{T}(-\mathbf{k})
\end{array}\right),
$$

where $\hat{\mathcal{H}}_{\eta}^{J, S, L}(\mathbf{k})$ is the pairing Hamiltonian in channel $(\eta, J, S, L)$ with $\eta$ being the relative basis label of the cubic irreducible representation (IR) [51,52]. The channel of instability is named by Cooper pair quantum numbers with a total angular momentum $J$ combining intrinsic spin $S$ and orbital $L$ angular momenta $[8,9,53]$.

To shed light on finite-energy pairing, the BdG excitation spectrum along the $[0,0,1]$ direction in the absence of spin-orbit coupling and pairing is plotted in Fig. 1(a). The fourfold degenerate electron bands (solid line) cross their hole counterparts (dashed line) at $k_{F}=\sqrt{\mu / \alpha}$. A finite $\beta$ accounting for spin-orbit coupling splits the energy bands having different magnetic quantum numbers. Increasing $\beta$, this moves the crossings at the Fermi surface $E=0$ and at FEEs (red circles), as shown in Fig. 1(b). The low-energy $m_{j}$ split Fermi momenta consist of $m_{j}=1 / 2$ states (green) and $m_{j}=$ $3 / 2$ states (black) located at $k_{F}^{-}=2 \sqrt{\mu /(4 \alpha+\beta)}$ and $k_{F}^{+}=$
$2 \sqrt{\mu /(4 \alpha+9 \beta)}$, respectively. Moreover, the finite-energy crossing appears at $\tilde{k}=2 \sqrt{\mu /(4 \alpha+5 \beta)}$ incorporating $m_{j}=$ $3 / 2$ electron (hole) and $m_{j}=1 / 2$ hole (electron) states at positive (negative) excitation energies. In the superconducting state, the pairing mechanism occurs not only at $E=0$ but also at FEEs [Figs. 1(c) and 1(d)]. Notably, the finiteenergy pairing can be present when the low-energy intraband states exhibit nodal [Fig. 1(c)] or gapped excitation spectra [Fig. 1(d)].

Finite-energy effective theory. To better understand the finite-energy pairing, we develop an effective theory close to the FEE. We start by obtaining the band basis representation of the BdG Hamiltonian through the basis transformation $\hat{c}_{\mathbf{k}}=\hat{V}_{\mathbf{k}}^{+} \hat{f}_{\mathbf{k}}^{+}+\hat{V}_{\mathbf{k}}^{-} \hat{f}_{\mathbf{k}}^{-}$, where $\hat{V}_{\mathbf{k}}^{ \pm}$is a $4 \times 2$ matrix containing the eigenvectors corresponding to $E_{\mathbf{k}}^{ \pm}$. Note that $\hat{f}_{\mathbf{k}}^{ \pm}=$ $\left(f_{\mathbf{k}, \uparrow}^{ \pm}, f_{\mathbf{k}, \downarrow}^{ \pm}\right)^{T}$ and $f_{\mathbf{k}, s}^{ \pm}\left(f_{\mathbf{k}, s}^{ \pm \dagger}\right)$ annihilates (creates) a state with pseudospin degrees of freedom $s \in\{\uparrow, \downarrow\}$ in the band basis labeled by \pm in Eq. (2). To capture the interband superconducting Hamiltonian, we choose our basis set as $\hat{\varphi}_{\mathbf{k}}=\left(\hat{\varphi}_{\mathbf{k}}^{+-}, \hat{\varphi}_{\mathbf{k}}^{-+}\right)^{T}$ with $\hat{\varphi}_{\mathbf{k}}^{+-}=\left(\hat{f}_{\mathbf{k}}^{+},\left(\hat{f}_{-\mathbf{k}}^{-\dagger}\right)^{T}\right)^{T}$ denoting the electron-hole subspace basis with band index $(+,-)$ and $\hat{\varphi}_{\mathbf{k}}^{-+}=\left(\hat{f}_{\mathbf{k}}^{-},\left(\hat{f}_{-\mathbf{k}}^{+\dagger}\right)^{T}\right)^{T}$. Thus, we rewrite the superconducting Hamiltonian in the band basis as $H=\sum_{\mathbf{k}} \hat{\varphi}_{\mathbf{k}}^{\dagger} \hat{h}(\mathbf{k}) \hat{\varphi}_{\mathbf{k}}$ with

$$
\hat{h}(\mathbf{k})=\left(\begin{array}{cccc}
E_{\mathbf{k}}^{+} & \hat{\Delta}_{\mathbf{k}}^{+-} & 0 & \hat{\Delta}_{\mathbf{k}}^{++} \\
\left(\hat{\Delta}_{\mathbf{k}}^{+-}\right)^{\dagger} & -E_{\mathbf{k}}^{-} & \left(\hat{\Delta}_{\mathbf{k}}^{--}\right)^{\dagger} & 0 \\
0 & \hat{\Delta}_{\mathbf{k}}^{--} & E_{\mathbf{k}}^{-} & \hat{\Delta}_{\mathbf{k}}^{-+} \\
\left(\hat{\Delta}_{\mathbf{k}}^{++}\right)^{\dagger} & 0 & \left(\hat{\Delta}_{\mathbf{k}}^{-+}\right)^{\dagger} & -E_{\mathbf{k}}^{+}
\end{array}\right),
$$

where $\hat{\Delta}_{\mathbf{k}}^{+-}$is the projection of the pairing instability onto the interband basis given by $\hat{\Delta}_{\mathbf{k}}^{+-}=\hat{V}_{\mathbf{k}}^{+\dagger} \hat{\mathcal{H}}_{\eta}^{J, S, L}(\mathbf{k})\left(\hat{V}_{-\mathbf{k}}^{-\dagger}\right)^{T}$. Treating the off-diagonal blocks, corresponding to the intraband pairing denoted by $\hat{\Delta}_{\mathbf{k}}^{v v}$ with $v \in\{+,-\}$, as a perturbation to the interband diagonal block and employing the folding down approach [54], we arrive at the effective Hamiltonian valid in the vicinity of the GLSs,

$$
H_{\text {eff }}^{+-}(\mathbf{k})=\left(\begin{array}{cc}
E_{\mathbf{k}}^{+}+\hat{\varepsilon}_{\mathbf{k}}^{++} & \hat{\Delta}_{\text {eff }}^{+-}(\mathbf{k}) \\
{\left[\hat{\Delta}_{\text {eff }}^{+-}(\mathbf{k})\right]^{\dagger}} & -E_{\mathbf{k}}^{-}+\hat{\varepsilon}_{\mathbf{k}}^{--}
\end{array}\right) .
$$

The second term on the diagonal in Eq. (5) is a pseudospin energy shift induced by the pairing of intraband quasiparticles, given by $\hat{\varepsilon}_{\mathbf{k}}^{v v}=\hat{\Delta}_{\mathbf{k}}^{v v}\left(\hat{\Delta}_{\mathbf{k}}^{v v}\right)^{\dagger} /\left(\omega+v E_{\mathbf{k}}^{v}\right)$. Notably, Eq. (5) is different from a typical $\mathrm{BdG}$ Hamiltonian. The effective particle-hole symmetry is broken due to the presence of nonidentical diagonal entries arising from the nature of two different energy bands. The interband pairing of the effective Hamiltonian takes the form

$$
\hat{\Delta}_{\text {eff }}^{+-}(\mathbf{k})=\hat{\Delta}_{\mathbf{k}}^{+-}+\varepsilon_{\mathbf{k}}^{-1} \hat{\Delta}_{\mathbf{k}}^{++}\left(\hat{\Delta}_{\mathbf{k}}^{-+}\right)^{\dagger} \hat{\Delta}_{\mathbf{k}}^{--},
$$

where $\varepsilon_{\mathbf{k}}=\left(\omega+E_{\mathbf{k}}^{+}\right)\left(\omega-E_{\mathbf{k}}^{-}\right)$[55]. In the weak-pairing limit, the second term is small close to the GLSs and can be neglected. The spectrum for the FEE reads

$$
\mathcal{E}_{ \pm}(\mathbf{k})=\varepsilon_{\mathbf{k}, 1}+\varepsilon_{\mathbf{k}, 2} \pm \sqrt{\left(\varepsilon_{\mathbf{k}, 1}-\varepsilon_{\mathbf{k}, 2}\right)^{2}+\stackrel{\wp}{\delta}(\mathbf{k})},
$$

where

$$
\stackrel{\circ}{\delta}(\mathbf{k})=\frac{1}{2} \operatorname{Tr}\left\{\hat{\Delta}_{\text {eff }}^{+-}(\mathbf{k})\left[\hat{\Delta}_{\text {eff }}^{+-}(\mathbf{k})\right]^{\dagger}\right\}
$$


TABLE I. Absence/presence of finite-energy Cooper pairing. The first column shows the IR of $O_{h}\left(T_{d}\right)$ point groups with $\eta$ denoting the basis label of the IR. The third column corresponds to the pairing multiplets of the relative IR. The last two columns indicate the presence $\checkmark$ (absence $\times$ ) of finite-energy Cooper pairing in the entire momentum space in the absence and presence of ASOC. The superscript $(*)$ means that $\delta(\mathbf{k})$ vanishes in all equivalent directions [62].

\begin{tabular}{lcccc}
\hline \hline$O_{h}\left(T_{d}\right)$ & $\eta$ & $(J, S, L)$ & Without ASOC & With ASOC \\
\hline$A_{1 g}\left(A_{1}\right)$ & $I$ & $(0,0,0)$ & $\times$ & $\times$ \\
$A_{1 u}\left(A_{2}\right)$ & $f(\boldsymbol{r})$ & $(0,1,1)$ & $\times$ & {$[1,1,1]^{*}$} \\
$T_{1 u}\left(T_{2}\right)$ & $z$ & $(1,1,1)$ & {$[0,0,1]$} & {$[0,0,1]$} \\
$E_{g}(E)$ & $3 z^{2}-r^{2}$ & $(2,2,0)$ & {$[0,0,1]$} & $\checkmark$ \\
& $x^{2}-y^{2}$ & $(2,2,0)$ & $\checkmark$ & $\checkmark$ \\
$E_{u}(E)$ & $3 z^{2}-r^{2}$ & $(2,1,1)$ & {$[0,0,1]^{*}, k_{z}=0$} & $\checkmark$ \\
& $3 z^{2}-r^{2}$ & $(2,3,1)$ & {$[0,0,1]$} & $\checkmark$ \\
& $x^{2}-y^{2}$ & $(2,1,1)$ & {$[0,0,1]^{*}$} & {$[0,0,1]$} \\
& $x^{2}-y^{2}$ & $(2,3,1)$ & $\checkmark$ & $\checkmark$ \\
$A_{2 u}\left(A_{1}\right)$ & $x y z$ & $(3,3,1)$ & {$[1,1,1]^{*}$} & {$[1,1,1]^{*}$} \\
\hline \hline
\end{tabular}

is the magnitude of the GLS indicating superconducting hybridization between interband states [56], i.e., pairing of $m_{j}=3 / 2$ with $m_{j}=1 / 2$ states; $\operatorname{Tr}$ stands for the trace of the matrix; $\varepsilon_{\mathbf{k}, 1}=(1 / 2) E_{\mathbf{k}}^{+}+(1 / 4) \operatorname{Tr}\left(\hat{\varepsilon}_{\mathbf{k}}^{++}\right)$and $\varepsilon_{\mathbf{k}, 2}=$ $-(1 / 2) E_{\mathbf{k}}^{-}+(1 / 4) \operatorname{Tr}\left(\hat{\varepsilon}_{\mathbf{k}}^{--}\right)$. The width of the GLSs around the finite-energy crossing momentum [57] is $\mid \mathcal{E}_{+}(\tilde{\boldsymbol{k}})-$ $\mathcal{E}_{-}(\tilde{\boldsymbol{k}}) \mid=2[\stackrel{\delta}{\delta}(\tilde{\boldsymbol{k}})]^{1 / 2}$. Note that the matrix form of $\hat{\Delta}_{\text {eff }}^{+-}(\mathbf{k}) \mathrm{de}-$ pends on the choice of basis while $\delta(\mathbf{k})$ is a basis-independent observable.

Symmetry properties. Interestingly, the symmetry properties of the finite-energy pairing are different from their low-energy counterpart. For instance, we may witness even (odd)-parity pseudospin triplet (singlet) pairing at FEE. This is a direct consequence of the Pauli exclusion principle taking into account the exchange of band indices in addition to the exchange of magnetic quantum numbers, i.e.,

$$
\hat{\Delta}_{\text {eff }}^{+-}(-\mathbf{k})=-\left[\hat{\Delta}_{\text {eff }}^{-+}(\mathbf{k})\right]^{T} .
$$

In this sense, we can span $\hat{\Delta}_{\text {eff }}^{+-}(\mathbf{k})$ in the interband basis as $\hat{\Delta}_{\text {eff }}^{+-}(\mathbf{k})=\mathfrak{g}^{+-}(\mathbf{k}) \cdot \boldsymbol{\tau}$, where the four-component vector $\mathfrak{g}^{+-}=\left(\mathfrak{g}_{0}^{+-}, \mathfrak{g}_{x}^{+-}, \mathfrak{g}_{y}^{+-}, \mathfrak{g}_{z}^{+-}\right)$is a complex momentumdependent function, $\boldsymbol{\tau}=\left(\tau_{0}, \tau_{x}, \tau_{y}, \tau_{z}\right)$ with $\tau_{x, y, z}$ being the Pauli matrices and $\tau_{0}$ the $2 \times 2$ identity matrix in the interband basis. Thus, we obtain the symmetry relations

$$
\mathfrak{g}_{0, x, z}^{+-}(-\mathbf{k})=-\mathfrak{g}_{0, x, z}^{-+}(\mathbf{k}), \quad \mathfrak{g}_{y}^{+-}(-\mathbf{k})=\mathfrak{g}_{y}^{-+}(\mathbf{k}) .
$$

This enables us to directly derive components of the $\hat{\Delta}_{\text {eff }}^{-+}(\mathbf{k})$. The $y$ component is even in momentum while the other components are odd [58].

Pairing channels of $\mathrm{O}_{h}$ symmetry. We apply our theory to all time-reversal symmetric stationary pairing states of cubic point group symmetry up to the $p$-wave channel $[9,59]$ with the aim to identify interband pairing. To obtain analytic relations for $\delta(\mathbf{k})$, we set $\gamma=\beta$ [60]. Note that the pairing states generate cubic anisotropy. The results are summarized in Table I. Remarkably, interband pairing is present for a variety of pairing channels.
First, we observe that the even- and odd-parity singlet pairing states [61], corresponding to the instability channels $A_{1 g}$ and $A_{1 u}$, respectively, have vanishing interband pairing, i.e., $\delta(\mathbf{k})=0$ [63]. Contrarily, the cubic triplet state $T_{1 u}[9,64]$ shows finite interband pairing $\delta(\mathbf{k})=\Delta^{2}\left(k_{x}^{2}+k_{y}^{2}\right)$ with $\Delta$ being the pairing strength. This indicates that the GLSs are present within the whole momentum space except for the $[0,0,1]$ direction where interband pairing vanishes. Next, we focus on pairing with quintet total angular momentum, i.e., $J=2$. In this case, the pairing state is split by the cubic field into $E_{g, u}+T_{2 g, u}$, where $E_{g, u}\left(T_{2 g, u}\right)$ is a two (three)dimensional IR. Note that the pairing state $E_{g, u}$ is a stationary state of the free energy whereas $T_{2 g, u}$ is not [9]. Hence, we focus on $E_{g, u}$ pairing in the following. The components of $E_{g, u}$ are denoted by $\eta=\left(3 z^{2}-r^{2}, x^{2}-y^{2}\right)$. In the $j=3 / 2$ representation, we find two (four) symmetry allowed pairing channels for even-parity (odd-parity) quintet pairing. For even-parity states, the quantum number is $(2,2,0)$, where the pairing Hamiltonian is momentum independent due to the $s$-wave nature of the channel. In this case, the GLSs of the $3 z^{2}-r^{2}$ state are given by $\delta(\mathbf{k})=3 \Delta_{\mathbf{k}}^{2}\left(k_{x}^{2}+k_{y}^{2}\right)\left(k^{2}+3 k_{z}^{2}\right)$ with $\Delta_{\mathbf{k}}=\Delta / 2 k^{2}$, showing nonvanishing GLSs except for the twofold rotation axis $[0,0,1]$. Importantly, the $x^{2}-y^{2}$ state exhibits full GLSs within the entire momentum space.

The odd-parity quintet channel has four momentumdependent stationary pairing states due to $L=1$. The first two states correspond to the $3 z^{2}-r^{2}$ basis having Cooper pair quantum numbers $(2,1,1)$ and $(2,3,1)$. These states differ only in the intrinsic spin quantum number where $S=1$ and $S=3$ denote spin dipole and octupole moments, respectively. The GLS for the former state takes the form $\delta(\mathbf{k})=27 \Delta_{k}^{2}\left(k_{x}^{2}+\right.$ $\left.k_{y}^{2}\right) k_{z}^{2}$ with $\Delta_{\mathbf{k}}=\Delta / 2 k$. It vanishes in the $[0,0,1]^{*}$ direction [62] as well as the $k_{z}=0$ plane. For the $S=3$ channel, the GLS becomes

$$
\grave{\delta}(\mathbf{k})=\Delta_{\mathbf{k}}^{2} \sum_{i}\left[\zeta_{i}^{(1)} k_{i}^{4}+\zeta_{i}^{(2)} k_{i}^{2} k_{i+1}^{2}\right],
$$

with $\zeta^{(1)}=(25,25,0)$ and $\zeta^{(2)}=(50,64,64)$. In this case, $\delta(\mathbf{k})$ is present in the entire momentum space except for the $z$ axis.

The GLS for the $p$-wave $x^{2}-y^{2}$ state in both $S=1(S=$ 3 ) channels can also be described by Eq. (11) with coefficients $\zeta^{(1)}=(0,0,0)$ and $\zeta^{(2)}=(4,1,1)\left[\zeta^{(1)}=(25 / 4,25 / 4,25)\right.$, $\left.\zeta^{(2)}=(103 / 2,41,41)\right]$. Hence, the $S=3$ channel demonstrates fully GLSs while the $S=1$ channel exhibits vanishing $\AA(\mathbf{k})$ along the $[0,0,1]^{*}$ direction.

Finally, we look at the septet state denoted by $A_{2 u}$. In this case, the pairing of electrons with different quantum numbers $m_{j}$ manifests itself by

$$
\grave{\delta}(\mathbf{k})=\frac{3 \Delta_{\mathbf{k}}^{2}}{16}\left\{\sum_{i}\left[4 k_{i}^{6}-3\left(k_{i}^{4} k_{i+1}^{2}+k_{i}^{4} k_{i+2}^{2}\right)\right]+6 k_{x}^{2} k_{y}^{2} k_{z}^{2}\right\},
$$

where $i+2=z$ if $i=x$ and the GLSs are present throughout the momentum space except for the $[1,1,1]^{*}$ direction.

Candidate systems with $T_{d}$ structure. It is worthwhile to note that the half-Heusler compounds $R \mathrm{PdBi}$ have tetrahedral $T_{d}$ symmetry (subgroup of $O_{h}$ ) without an inversion center. Nevertheless, the formalism of describing the pairing is the 


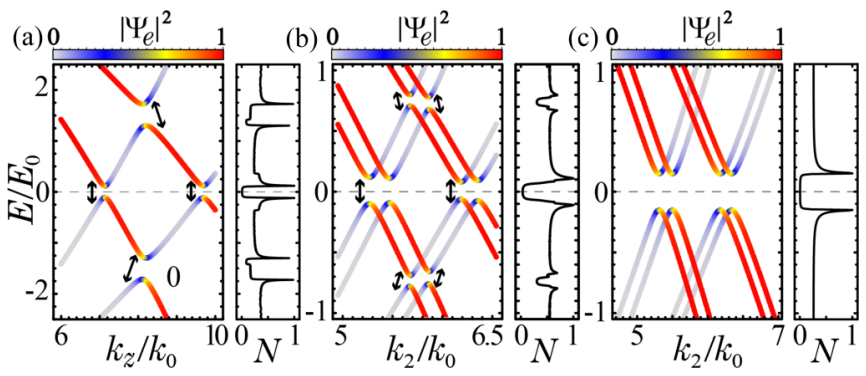

FIG. 2. BdG spectra in (a) $[0,0,1]$ and (b), (c) $[1,0,1]$ (with $k_{x}=$ $\left.k_{z}=k_{2}\right)$ directions for (a) $\left(\Delta / E_{0} a, \delta / E_{0} a\right)=(3.5,10)$, (b) $(1.5,2.5)$, and (c) $(0.15,2.5)$, respectively. (a) and (b) correspond to septet pairing, and (c) corresponds to $A_{1 g}$ pairing. The corresponding density of states $N$ normalized with respect to its maximum value are presented in the right panel of each spectrum. $\gamma=-0.05|\alpha|$ and other parameters are the same as those in Fig. 1.

same as for the $O_{h}$ group but different IR labels apply (cf. Table I). The noncentrosymmetry manifests itself by an antisymmetric spin-orbit coupling (ASOC) given by $[6,65]$

$$
\hat{H}^{\prime}(\mathbf{k})=\delta \sum_{i} k_{i}\left(\hat{J}_{i+1} \hat{J}_{i} \hat{J}_{i+1}-\hat{J}_{i+2} \hat{J}_{i} \hat{J}_{i+2}\right),
$$

where $\delta$ controls the strength of the ASOC and $i \in\{x, y, z\}$. Projecting $\hat{H}^{\prime}(\mathbf{k})$ onto the intraband basis, this results in splitting the energy band as $E_{\mathbf{k}}^{v} \rightarrow E_{\mathbf{k}}^{v} \pm\left|\boldsymbol{g}_{\mathbf{k}}^{\nu v}\right|$ with $\boldsymbol{g}_{\mathbf{k}}^{\nu v}$. $\sigma=\hat{V}_{\mathbf{k}}^{\nu \dagger} \hat{H}^{\prime}(\mathbf{k}) \hat{V}_{\mathbf{k}}^{\nu}$ and $v= \pm$, as shown in Figs. 2(b) and 2(c) [66]. Here, $\boldsymbol{g}_{\mathbf{k}}^{\nu v}=\left(g_{x}^{\nu v}, g_{y}^{\nu v}, g_{z}^{v v}\right)$ and $\boldsymbol{\sigma}=\left(\hat{\sigma}_{x}, \hat{\sigma}_{y}, \hat{\sigma}_{z}\right)$ are momentum-dependent ASOC vector and Pauli matrices in the intraband basis, respectively. The lack of inversion symmetry allows the pairing state to be a mixture of even-parity singlet $\hat{\mathcal{H}}_{I}^{0,0,0}(\mathbf{k})$ and odd-parity $p$-wave states [67]. In this case, the most stable odd-parity pairing state with the largest transition temperature may arise when its $\mathbf{d}$ vector aligns parallel to the ASOC vector $[42,68]$. Thus, by combining $\hat{H}^{\prime}(\mathbf{k})$ with the Cooper pair symmetrization matrix $\hat{\mathcal{R}}=i \hat{\sigma}_{x} \otimes \hat{\sigma}_{y}$ in the $j=3 / 2$ representation, we arrive at the septet pairing state $\hat{\mathcal{H}}_{x y z}^{3,3,1}(\mathbf{k})=\hat{H}^{\prime}(\mathbf{k}) \hat{\mathcal{R}}$ [6]. The interband crossing of the mixed superconducting state $\hat{\mathcal{H}}_{I}^{0,0,0}(\mathbf{k})+\hat{\mathcal{H}}_{x y z}^{3,3,1}(\mathbf{k})$ cannot be hybridized by the inversion symmetry breaking ASOC.
Therefore, the emergence of finite-energy superconducting coherence peaks in the DOS are strong indicators of septet Cooper pairing of electrons with different quantum numbers $m_{j}$, as shown in Figs. 2(a) and 2(b). Note the difference from singlet pairing, where the DOS exhibit a flat shape away from the Fermi surface [cf. Fig. 2(c)]. Remarkably, both odd- and even-parity channels of $3 z^{2}-r^{2}$ turn into fully GLSs in the presence of ASOC (cf. Table I). This also partially happens for the $A_{2}$ state and the $\left(x^{2}-y^{2}, 2,1,1\right)$ state. Therefore, a small value of ASOC even enhances the likelihood of observing GLSs in the DOS.

To observe the $m_{j}$ content of the pairing at FEE, we propose to apply a perturbative Zeeman field to the system where the states acquire finite magnetization in terms of $m_{j}$ degrees of freedom due to broken time-reversal symmetry [53]. Consequently, the GLSs split into two different pairs of GLSs. Each GLS corresponds to paired electrons with different magnetic quantum numbers signaled by simultaneous drops in the $m_{j}$-resolved DOS.

Conclusions. We have investigated Cooper pairing in $j=$ $3 / 2$ superconductors with cubic point group symmetry. The multiband nature of the system with an identical bending configuration allows for observing Cooper pairing away from the Fermi surface in the weak pairing limit. This manifests itself by a pair of indirect finite-energy anticrossings of $\mathrm{BdG}$ bands signaling the pairing of electrons having different components of total angular momentum. The phenomenon may be experimentally detectable through tunneling spectroscopy [69-72] and angle-resolved photoemission spectroscopy [73].

Note added. Recently, we became aware of a related proposal of interband pairing away from the Fermi surface. This proposal is about the emergence of anapole superconductivity in the presence of competing pairing channels. Hence, the physics is different from ours [74].

Acknowledgments. We thank M. Bode, S. J. Choi, P. Eck, M. V. Hosseini, C. A. Li, G. Sangiovanni, and A. H. Talebi for fruitful discussions. The work was supported by the DFG (SPP1666 and SFB1170 ToCoTronics), the WürzburgDresden Cluster of Excellence ct.qmat, EXC2147, Project Id 390858490, and the Elitenetzwerk Bayern Graduate School on Topological Insulators.
[1] J. Bardeen, L. N. Cooper, and J. R. Schrieffer, Theory of superconductivity, Phys. Rev. 108, 1175 (1957).

[2] J. P. Carbotte, Properties of boson-exchange superconductors, Rev. Mod. Phys. 62, 1027 (1990).

[3] M. Sigrist and K. Ueda, Phenomenological theory of unconventional superconductivity, Rev. Mod. Phys. 63, 239 (1991).

[4] T.-L. Ho and S. Yip, Pairing of Fermions with Arbitrary Spin, Phys. Rev. Lett. 82, 247 (1999).

[5] W. Yang, Y. Li, and C. Wu, Topological Septet Pairing with Spin- $\frac{3}{2}$ Fermions: High-Partial-Wave Channel Counterpart of the ${ }^{3} \mathrm{He}-B$ Phase, Phys. Rev. Lett. 117, 075301 (2016).

[6] P. M. R. Brydon, L. Wang, M. Weinert, and D. F. Agterberg, Pairing of $j=3 / 2$ Fermions in Half-Heusler Superconductors, Phys. Rev. Lett. 116, 177001 (2016).
[7] V. Kozii, J. W. F. Venderbos, and L. Fu, Three-dimensional Majorana fermions in chiral superconductors, Sci. Adv. 2, 12 (2016).

[8] L. Savary, J. Ruhman, J. W. F. Venderbos, L. Fu, and P. A. Lee, Superconductivity in three-dimensional spin-orbit coupled semimetals, Phys. Rev. B 96, 214514 (2017).

[9] J. W. F. Venderbos, L. Savary, J. Ruhman, P. A. Lee, and L. Fu, Pairing States of Spin- $\frac{3}{2}$ Fermions: SymmetryEnforced Topological Gap Functions, Phys. Rev. X 8, 011029 (2018).

[10] P. Dutta, F. Parhizgar, and A. M. Black-Schaffer, Superconductivity in spin-3/2 systems: Symmetry classification, odd-frequency pairs, and Bogoliubov Fermi surfaces, Phys. Rev. Research 3, 033255 (2021). 
[11] C. Fang, B. A. Bernevig, and M. J. Gilbert, Tri-Dirac surface modes in topological superconductors, Phys. Rev. B 91, 165421 (2015).

[12] I. Boettcher and I. F. Herbut, Superconducting quantum criticality in three-dimensional Luttinger semimetals, Phys. Rev. B 93, 205138 (2016).

[13] D. F. Agterberg, P. M. R. Brydon, and C. Timm, Bogoliubov Fermi Surfaces in Superconductors with Broken Time-Reversal Symmetry, Phys. Rev. Lett. 118, 127001 (2017).

[14] C. Timm, A. P. Schnyder, D. F. Agterberg, and P. M. R. Brydon, Inflated nodes and surface states in superconducting half-Heusler compounds, Phys. Rev. B 96, 094526 (2017).

[15] J. Yu and C.-X. Liu, Singlet-quintet mixing in spin-orbit coupled superconductors with $j=\frac{3}{2}$ fermions, Phys. Rev. B 98, 104514 (2018).

[16] T. Kawakami, T. Okamura, S. Kobayashi, and M. Sato, Topological Crystalline Materials of $J=3 / 2$ Electrons: Antiperovskites, Dirac Points, and High Winding Topological Superconductivity, Phys. Rev. X 8, 041026 (2018).

[17] S. Kobayashi, A. Yamakage, Y. Tanaka, and M. Sato, Majorana Multipole Response of Topological Superconductors, Phys. Rev. Lett. 123, 097002 (2019).

[18] B. Roy, Sayed Ali Akbar Ghorashi, M. S. Foster, and A. H. Nevidomskyy, Topological superconductivity of spin-3/2 carriers in a three-dimensional doped Luttinger semimetal, Phys. Rev. B 99, 054505 (2019).

[19] H. Menke, C. Timm, and P. M. R. Brydon, Bogoliubov Fermi surfaces stabilized by spin-orbit coupling, Phys. Rev. B 100, 224505 (2019).

[20] S.-T. Tamura, S. Iimura, and S. Hoshino, Electronic multipoles and multiplet pairs induced by Pomeranchuk and Cooper instabilities of Bogoliubov Fermi surfaces, Phys. Rev. B 102, 024505 (2020).

[21] C. Timm, P. M. R. Brydon, and D. F. Agterberg, Distortional weak-coupling instability of Bogoliubov Fermi surfaces, Phys. Rev. B 103, 024521 (2021).

[22] A. L. Szabó, R. Moessner, and B. Roy, Interacting spin- $\frac{3}{2}$ fermions in a Luttinger semimetal: Competing phases and their selection in the global phase diagram, Phys. Rev. B 103, 165139 (2021).

[23] G. Goll, M. Marz, A. Hamann, T. Tomanic, K. Grube, T. Yoshino, and T. Takabatake, Thermodynamic and transport properties of the non-centrosymmetric superconductor LaBiPt, Phys. B: Condens. Matter 403, 1065 (2008).

[24] H. Lin, L. A. Wray, Y. Xia, S. Xu, S. Jia, R. J. Cava, A. Bansil, and M. Z. Hasan, Half-Heusler ternary compounds as new multifunctional experimental platforms for topological quantum phenomena, Nat. Mater. 9, 546 (2010).

[25] S. Chadov, X. Qi, J. Kübler, G. H. Fecher, C. Felser, and S. C. Zhang, Tunable multifunctional topological insulators in ternary Heusler compounds, Nat. Mater. 9, 541 (2010).

[26] N. P. Butch, P. Syers, K. Kirshenbaum, A. P. Hope, and J. Paglione, Superconductivity in the topological semimetal YPtBi, Phys. Rev. B 84, 220504(R) (2011).

[27] T. V. Bay, T. Naka, Y. K. Huang, and A. de Visser, Superconductivity in noncentrosymmetric YPtBi under pressure, Phys. Rev. B 86, 064515 (2012).

[28] F. F. Tafti, T. Fujii, A. Juneau-Fecteau, S. R. de Cotret, N. Doiron-Leyraud, A. Asamitsu, and L. Taillefer, Superconductivity in the noncentrosymmetric half-Heusler compound
LuPtBi: A candidate for topological superconductivity, Phys. Rev. B 87, 184504 (2013).

[29] Y. Pan, A. M. Nikitin, T. V. Bay, Y. K. Huang, C. Paulsen, B. H. Yan, and A. de Visser, Superconductivity and magnetic order in the noncentrosymmetric half-Heusler compound ErPdBi, Europhys. Lett. 104, 27001 (2013).

[30] G. Xu, W. Wang, X. Zhang, Y. Du, E. Liu, S. Wang, G. Wu, Z. Liu, and X. X. Zhang, Weak antilocalization effect and noncentrosymmetric superconductivity in a topologically nontrivial semimetal LuPdBi, Sci. Rep. 4, 5709 (2014).

[31] Y. Nakajima, R. Hu, K. Kirshenbaum, A. Hughes, P. Syers, X. Wang, K. Wang, R. Wang, S. R. Saha, D. Pratt, J. W. Lynn, and J. Paglione, Topological RPdBi half-Heusler semimetals: A new family of noncentrosymmetric magnetic superconductors, Sci. Adv. 1, e1500242 (2015).

[32] A. M. Nikitin, Y. Pan, X. Mao, R. Jehee, G. K. Araizi, Y. K. Huang, C. Paulsen, S. C. Wu, B. H. Yan, and A. de Visser, Magnetic and superconducting phase diagram of the half-Heusler topological semimetal HoPdBi, J. Phys.: Condens. Matter 27, 275701 (2015)

[33] Z. K. Liu, L. X. Yang, S.-C. Wu, C. Shekhar, J. Jiang, H. F. Yang, Y. Zhang, S.-K. Mo, Z. Hussain, B. Yan, C. Felser, and Y. L. Chen, Observation of unusual topological surface states in half-Heusler compounds LnPtBi $(\mathrm{Ln}=\mathrm{Lu}, \mathrm{Y})$, Nat. Commun. 7, 12924 (2016).

[34] M. Meinert, Unconventional Superconductivity in YPtBi and Related Topological Semimetals, Phys. Rev. Lett. 116, 137001 (2016).

[35] D. Shrivastava and S. P. Sanyal, Electronic structure and superconducting behaviour of LuPtBi half-Heusler compound: A first principle study, Physica C 544, 22 (2018).

[36] H. Kim, K. Wang, Y. Nakajima, R. Hu, S. Ziemak, P. Syers, L. Wang, H. Hodovanets, J. D. Denlinger, P. M. R. Brydon, D. F. Agterberg, M. A. Tanatar, R. Prozorov, and J. Paglione, Beyond triplet: Unconventional superconductivity in a spin-3/2 topological semimetal, Sci. Adv. 4, 4 (2018).

[37] H. Xiao, T. Hu, W. Liu, Y. L. Zhu, P. G. Li, G. Mu, J. Su, K. Li, and Z. Q. Mao, Superconductivity in the half-Heusler compound TbPdBi, Phys. Rev. B 97, 224511 (2018).

[38] S. M. A. Radmanesh, C. Martin, Y. Zhu, X. Yin, H. Xiao, Z. Q. Mao, and L. Spinu, Evidence for unconventional superconductivity in half-Heusler YPdBi and TbPdBi compounds revealed by London penetration depth measurements, Phys. Rev. B 98, 241111(R) (2018).

[39] R. Majumder and M. M. Hossain, First-principles study of structural, electronic, elastic, thermodynamic and optical properties of topological superconductor LuPtBi, Comput. Condens. Matter 21, e00402 (2019).

[40] M. M. Hosen, G. Dhakal, K. Dimitri, H. Choi, F. Kabir, C. Sims, O. Pavlosiuk, P. Wiśniewski, T. Durakiewicz, J.-X. Zhu, D. Kaczorowski, and M. Neupane, Observation of Dirac state in half-Heusler material YPtBi, Sci. Rep. 10, 12343 (2020).

[41] V. Bhardwaj, A. Bhattacharya, S. Srivastava, V. V. Khovaylo, J. Sannigrahi, N. Banerjee, B. K. Mani, and R. Chatterjee, Strain driven emergence of topological non-triviality in YPdBi thin films, Sci. Rep. 11, 7535 (2021).

[42] K. Ishihara, T. Takenaka, Y. Miao, Y. Mizukami, K. Hashimoto, M. Yamashita, M. Konczykowski, R. Masuki, M. Hirayama, T. Nomoto, R. Arita, O. Pavlosiuk, P. Wisniewski, D. Kaczorowski, and T. Shibauchi, Tuning the Parity Mixing of 
Singlet-Septet Pairing in a Half-Heusler Superconductor, Phys. Rev. X 11, 041048 (2021).

[43] W. Al-Sawai, H. Lin, R. S. Markiewicz, L. A. Wray, Y. Xia, S.-Y. Xu, M. Z. Hasan, and A. Bansil, Topological electronic structure in half-Heusler topological insulators, Phys. Rev. B 82, 125208 (2010).

[44] A. Jain, S. P. Ong, G. Hautier, W. Chen, W. D. Richards, S. Dacek, S. Cholia, D. Gunter, D. Skinner, G. Ceder, and K. A. Persson, Commentary: The Materials Project: A materials genome approach to accelerating materials innovation, APL Mater. 1, 011002 (2013).

[45] C. Shi, X. Xi, Z. Hou, X. Zhang, G. Xu, E. Liu, W. Wang, W. Wang, J. Chen, and G. Wu, NMR investigation of atomic and electronic structures of half-Heusler topologically nontrivial semimetals, Phys. Status Solidi B 252, 357 (2015).

[46] For $R \in\{\mathrm{Y}, \mathrm{Dy}, \mathrm{Tb}, \mathrm{Sm}\}$ in $R \mathrm{PdBi}$, the downward curving $m_{j}$ split $\Gamma_{8}$ bands lie close to the Fermi surface. This can be clearly seen for YPdBi in Refs. [24,25,41,43-45] and for $R \in$ $\{\mathrm{Dy}, \mathrm{Tb}, \mathrm{Sm}\}$ in Ref. [44].

[47] A. Moreo, M. Daghofer, A. Nicholson, and E. Dagotto, Interband pairing in multiorbital systems, Phys. Rev. B 80, 104507 (2009).

[48] G. Tang, C. Bruder, and W. Belzig, Magnetic Field-Induced "Mirage" Gap in an Ising Superconductor, Phys. Rev. Lett. 126, 237001 (2021).

[49] J. M. Luttinger and W. Kohn, Motion of electrons and holes in perturbed periodic fields, Phys. Rev. 97, 869 (1955).

[50] G. Dresselhaus, Spin-orbit coupling effects in zinc blende structures, Phys. Rev. 100, 580 (1955).

[51] M. Tinkham, Group Theory and Quantum Mechanics, Dover Books on Chemistry and Earth Sciences (Dover, New York, 2003).

[52] M. Dresselhaus, G. Dresselhaus, and A. Jorio, Group Theory: Application to the Physics of Condensed Matter (Springer, Berlin, 2007).

[53] See Supplemental Material at http://link.aps.org/supplemental/ 10.1103/PhysRevResearch.4.L012017 for the details on the folding down approach, power series expansion, band basis formalism, calculation of angular-momentum-resolved density of states, detection of the $m_{j}$ composition of Cooper pairs, spectrum of $j=3 / 2$ pairing at FEE, and constructing pairing Hamiltonians in cubic point group symmetry, which includes Refs. $[6,8,9,15,20,42,51,52,54,64]$.

[54] P. Löwdin, A note on the quantum-mechanical perturbation theory, J. Chem. Phys. 19, 1396 (1951).

[55] The analogous model for the $(-+)$ subspace can be derived by substituting $(+) \leftrightarrow(-)$ in Eq. (5).

[56] Due to our choice of the pairing state, the interband gaplike structure $\delta(\mathbf{k})$ is proportional to the identity matrix.

[57] The band crossings of the FEE appear for the $C_{2}, C_{2}^{\prime}$, and $C_{3}$ axes at $E= \pm 4|\mu \beta /(4 \alpha+5 \beta)|, E= \pm 2 \mid \mu /(4 \alpha+$ $5 \beta) \mid \sqrt{\beta^{2}+3 \gamma^{2}}$, and $E= \pm 4|\gamma \mu /(4 \alpha+5 \beta)|$, respectively. These crossings constitute the prerequisite for the GLSs.

[58] In the case of low-energy pairing of intraband electrons, the usual even (odd)-parity pseudospin singlet (triplet) as well as the $\boldsymbol{d}$-vector representation of the odd-parity states $[3,75]$ can be deduced from $\hat{\Delta}_{\text {eff }}^{v v^{\prime}}(-\mathbf{k})=-\left[\hat{\Delta}_{\text {eff }}^{v v^{\prime}}(\mathbf{k})\right]^{T}$ with $v=v^{\prime} \in\{+,-\}$.
[59] We expand the electron-electron interaction through the orthogonal basis set of cubic point group symmetry. In this regard, the expanded two-body interaction includes many terms, each distinguished by the basis label of cubic symmetry and Cooper pair quantum numbers. We investigate each term individually.

[60] For cubic anisotropy in the normal-state $\gamma \neq \beta$, our results are still valid.

[61] The pairing channel nomenclature is based on the $J$ quantum number of Cooper pairs due to the presence of strong spin-orbit coupling.

[62] There are three (eight) equivalent directions for the proper twofold (threefold) rotation $C_{2(3)}$ in cubic point group symmetry. The equivalent directions for the $C_{2}$ rotation are $[1,0,0]$, $[0,1,0]$, and $[0,0,1]$. For the $C_{3}$ rotation, the equivalent directions are $[1,1,1],[\overline{1}, 1,1],[1, \overline{1}, 1],[1,1, \overline{1}],[\overline{1}, \overline{1}, 1],[1, \overline{1}, \overline{1}]$, $[\overline{1}, 1, \overline{1}]$, and $[\overline{1}, \overline{1}, \overline{1}]$, where $\overline{1} \equiv-1$.

[63] $g(u)$ stands for even (odd) parity depending on the symmetry of the Cooper pair quantum numbers.

[64] H. Mäkelä and K.-A. Suominen, Inert States of Spin-S Systems, Phys. Rev. Lett. 99, 190408 (2007).

[65] W. Yang, T. Xiang, and C. Wu, Majorana surface modes of nodal topological pairings in spin- $\frac{3}{2}$ semimetals, Phys. Rev. B 96, 144514 (2017).

[66] In Fig. 2(a), the twofold degeneracy along the $[0,0,1]^{*}$ direction is not lifted in the presence of the inversion breaking ASOC term. This degeneracy is protected by mirror reflection symmetry and can be witnessed in time-reversal symmetric $T_{d}$ crystals [65].

[67] E. Bauer and M. Sigrist, Non-Centrosymmetric Superconductors: Introduction and Overview, Lecture Notes in Physics (Springer, Berlin, 2012), Vol. 847.

[68] P. A. Frigeri, D. F. Agterberg, A. Koga, and M. Sigrist, Superconductivity without Inversion Symmetry: MnSi versus $\mathrm{CePt}_{3} \mathrm{Si}$, Phys. Rev. Lett. 92, 097001 (2004).

[69] T. Dvir, F. Massee, L. Attias, M. Khodas, M. Aprili, C. H. L. Quay, and H. Steinberg, Spectroscopy of bulk and few-layer superconducting $\mathrm{NbSe}_{2}$ with van der Waals tunnel junctions, Nat. Commun. 9, 598 (2018).

[70] Y. Okada, Y. Ando, R. Shimizu, E. Minamitani, S. Shiraki, S. Watanabe, and T. Hitosugi, Scanning tunnelling spectroscopy of superconductivity on surfaces of $\mathrm{LiTi}_{2} \mathrm{O}_{4}(111)$ thin films, Nat. Commun. 8, 15975 (2017).

[71] R. Kumar, A. Vasdev, S. Das, S. Howlader, K. S. Jat, P. Neha, S Patnaik, and G. Sheet, The pressure-enhanced superconducting phase of $\mathrm{Sr}_{x}-\mathrm{Bi}_{2} \mathrm{Se}_{3}$ probed by hard point contact spectroscopy, Sci. Rep. 11, 4090 (2021).

[72] D. Costanzo, H. Zhang, B. A. Reddy, H. Berger, and A. F. Morpurgo, Tunnelling spectroscopy of gate-induced superconductivity in $\mathrm{MoS}_{2}$, Nat. Nanotechnol. 13, 483 (2018).

[73] M. Hashimoto, I. M. Vishik, R.-H. He, T. P. Devereaux, and Z.-X. Shen, Energy gaps in high-transition-temperature cuprate superconductors, Nat. Phys. 10, 483 (2014).

[74] S. Kanasugi and Y. Yanase, Anapole superconductivity from $\mathcal{P} \mathcal{T}$-symmetric mixed-parity interband pairing, arXiv:2107.07096.

[75] R. Balian and N. R. Werthamer, Superconductivity with pairs in a relative $p$ wave, Phys. Rev. 131, 1553 (1963). 